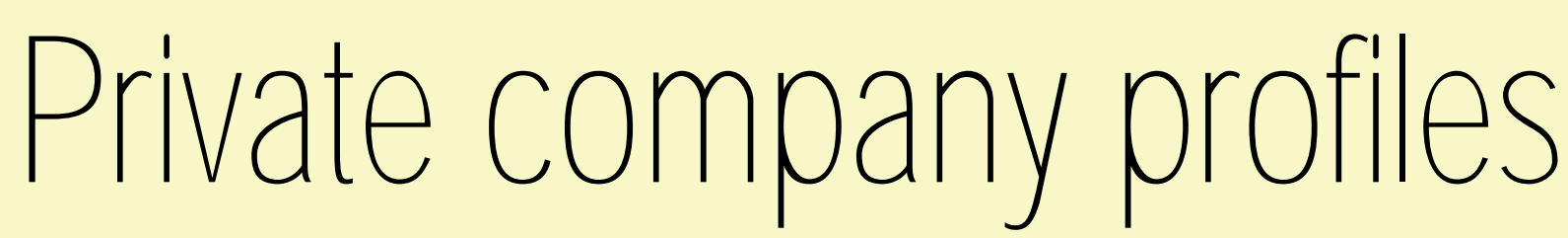

This section profiles a select group of private companies that are in the business of developing cutting-edge technologies. Companies were selected on the basis of the track record of their founding scientists, the novelty and breadth of application of their technologies, and potential for access to defined and lucrative target markets.

\title{
Cell Based Delivery
}

\author{
Engineering muscle cells for implantable drug delivery.
}

Founded: June 1997

Founders: Robert F. Valentini;

Herman H. Vandenburgh.

Employees: 15

Financing to date: $\$ 6$ million, including private investment $(\$ 500,000)$ and grants, awards, and contracts from: National Institute of Standards and Technology, Advanced Technology Program Small Business Innovation Research National Institutes of Health Defense Advance Research Program Agency

Location: Providence, RI

http://www.cbd-biotech.com

Startup Profiles written by Aaron Bouchie, Emma Dorey, Liz Fletcher, and M ichael Francisco. ost biotechnology products today are biopharmaceuticals- proteins, antibodies, hormones, growth factors, or enzymeswhich are expensive, difficult to manufacture, and require frequent injection because they are quickly degraded by the body. Cell Based Delivery hopes to overcome these limitations by- as its name suggests- harnessing a cell's ability to manufacture and deliver biotherapeutics.

Cell Based Delivery engineers human cells and tissues- primarily from skeletal muscle - to becomeliving drug-delivery systems, through its so-called ImPACT (implantable protein factory) system. The company introduces genes encoding therapeutic proteins into human muscle stem cells, fibroblasts, or other cell types- either from the patient directly or from donor cell banks- and then organizes the cells into compact implants about $20 \mathrm{~mm}$ long and 1-2 $\mathrm{mm}$ in diameter. This procedure is carried out in vitro, so the company can gauge how much protein is released from the engineered tissue. The tissue is then implanted in the body, using standard procedures, where it delivers a controlled and sustained dose of therapeutic protein.

ImPACT products have survived in animals for up to six months with no sign of tissue loss, indicating that long-lasting drug delivery can beachieved with just a single procedure. Currently available proteindelivery systems, such as biodegradable microspheres, provide only one to two weeks of continuous dosing.

Cell Based Delivery expects that its initial products will be generated from a patient's own cells (autologous), avoiding problems associated with the rejection of transplanted donor tissues. M oreover, because muscle cells live for years, autologous ImPACT products could ultimately last a year or longer. Allogeneic products (from donor cells) are reabsorbed over time and so are being developed for shorter-term protein delivery.

Robert Valentini, Cell Based Delivery's chief executive officer, says that I mPACT products can be used to treat a broad range of cardiovascular or chronic diseases. For example, ImPACT products placed in or around the heart could beused to deliver angiogenic factors to ischemic heart tissue. Products placed below the skin could deliver proteins systemical ly for the treatment of, for example, chronic diseases such as hemophilia or anemia. ImPACT products have been created to deliver vascular endothelial growth factor, erythropoietin (EPO), growth hormone, and insulin-likegrowth factor 1.

The company has a two-pronged business strategy: First, Cell Based Delivery will partner its therapeutic angiogenesis ImPACT products with leading pharmaceutical and biotechnology companies, in exchange for up-front fees, research funding, milestones, and royalties on future product sales. Second, the company is developing its own products based on proteins- such as factor VIII (for hemophilia) and EPO (for anemia) - approved by the US Food and Drug Administration (Rockville, MD) but soon to lose patent protection, for independent commercialization or co-development. Valentini says that Cell Based Delivery is in discussions with potential investors and corporate partners, and expects to begin clinical testing within 18 months. 